\title{
Event reconstruction in the BM@N experiment
}

\author{
P. Batyuk ${ }^{1, *}, D$. Baranov $^{1}, S$. Merts $^{1}$, and $O$. Rogachevsky ${ }^{1}$ \\ ${ }^{1}$ Veksler and Baldin Laboratory of High Energy Physics, JINR, 141980 Dubna, Russia
}

\begin{abstract}
In the paper, the main accent is put on development of software to be used in the BM@N experiment. The experiment is considered as a first step towards a realization of fixed target program at the NICA complex. A brief description of software used for reconstruction of track parameters in the inner tracker of the experiment is presented. The alignment procedure in automatic mode was made using the Millepede package fully integrated in the software. The obtained results illustrate a quality assurance of alignment performed using existing experimental data from experimental runs and recent results, including methodological ones from a tracking procedure, which were used for event reconstruction in the inner tracker. The importance of a precise geometry description and a realistic detector response via micro-simulations done for the GEM part of the inner tracker used for processing experimental data is emphasized. Our recent progress in this activity is discussed.
\end{abstract}

\section{Experimental setup}

BM@N (Baryonic matter at Nuclotron) [1] is considered as a first step towards realization of physics program at the NICA complex. It covers a fixed target program available at Nuclotron with extracted beams of different species. The experiment performed a set of technical runs mainly aimed to test sub-detector systems. The last one took place in the spring of 2018 using argon and krypton beams available in a range of kinetic energies of $2.3-3.2 \mathrm{AGeV}$.

Schematic view of the experimental setup is shown in Fig. 1. In the paper, the main accent is put on development of reconstruction algorithms in relation to GEM (n.4 in Fig. 1) and silicon detectors (n.3 in Fig. 1). The GEM and silicon detectors form together the inner tracker system of the experiment. It consists of nine sensitive planes, in particular, three of them correspond to a silicon detector and the other six - to GEM.

\section{Reconstruction algorithm for the inner tracker}

The reconstruction of spatial coordinates of points produced by a charge particle in a sensitive element of a detector serves as a necessary step towards finding tracks and estimation of their parameters. These points are called "hits".

The algorithm to be used for reconstruction of hits consists of two subsequent stages: clusterization and search of hits by strip intersections.

The detectors for track reconstruction used in the inner tracker of the BM@N experiment have a microstrip readout. It is a two-layer structure of strip assembly used to calculate

\footnotetext{
*e-mail: pavel.batyuk@jinr.ru
} 


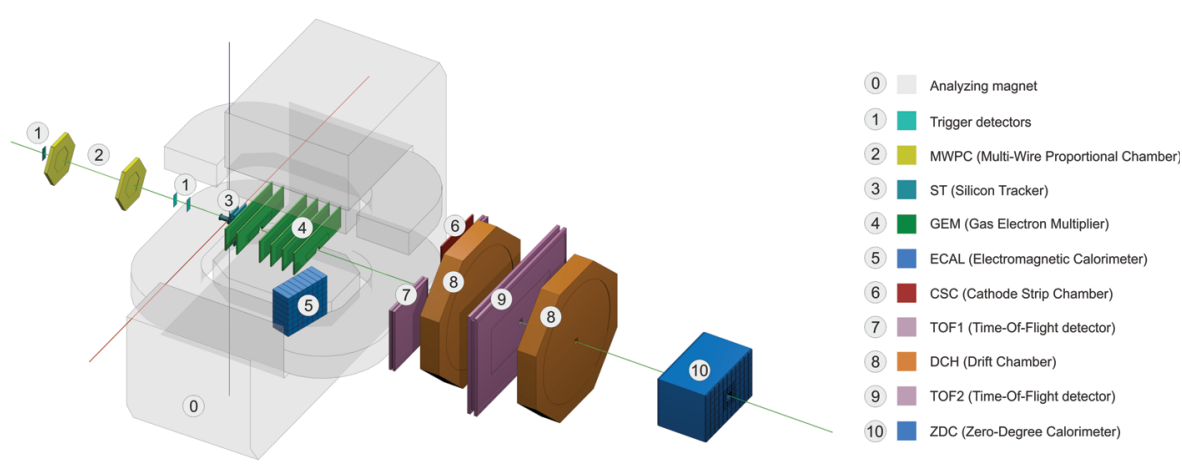

Figure 1. View of the experimental setup in the last run

spatial coordinates represented by intersections of fired strips. Search for a cluster and its parameterization are done separately in each layer. Generally speaking, a layer with a given set of fired strips is considered as a one-dimensional discrete spectrum to be analyzed. In course of this analysis one has to reveal cluster structures of peak form in the spectrum. The found clusters are thus parametrized giving us a set of parameters which characterize each cluster. The most important among them are position of center of gravity of the cluster along strip axis, width of the cluster and its total charge. The positions of center found in two layers at the previous step describe average positions of fired strips to be intersected in order to get spatial coordinates of a hit (see Fig. 2).

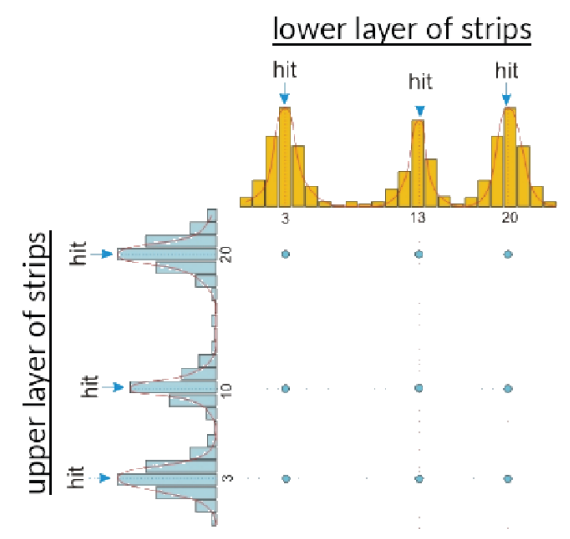

Figure 2. Schematic interpretation of the reconstruction algorithm

The procedure described above is enhanced by additional post-processing algorithms aiming at noise filtering, unfolding of clusters located in close proximity, etc.

Another important point to be stressed is a fact that the use of detector planes based on the strip readout has a big disadvantage revealing itself when obtaining a set of false intersections called "fakes" (see Fig. 3). There are different ways to suppress them. It can be achieved on a detector construction stage as well as when doing a program post-processing of existing data. Firstly, the readout plane of detector is divided into small zones with its own set of strips. The 


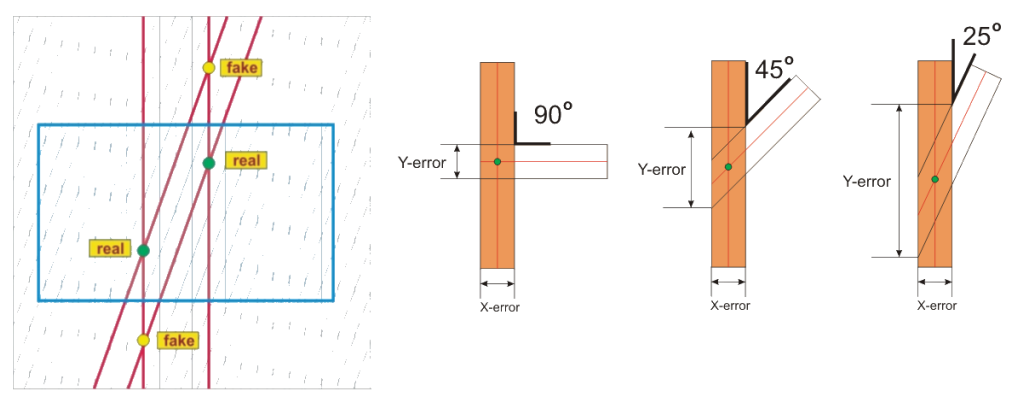

Figure 3. Illustration of "fake" production

smaller the length of the strip, the less the number of intersections obtained with other strips. Secondly, to prevent production of "fakes", the strips of two layers have a relatively small angle of intersection (stereo angle). In particular, the GEM detectors that we are using have a stereo angle of order of 15 degrees. At the same time, a value of stereo angle for silicon detectors of the inner tracker is equal to 2.5 degrees. It should be stressed that decreasing the stereo angle too much leads to a big uncertainty when reconstructing Y-coordinate. A possible solution to reduce the uncertainty follows pitch decrease (distance between strips), but it leads to the increase of number of channels of electronics and complication of detector. Presently, the pitch values are $800 \mu m$ for GEM and of order of $100 \mu m$ for the silicon detector. A program post-processing aiming at preventing production of "fakes" takes place when doing signal filtering. It is necessary to suppress the noise as much as possible, to mark strips that do not work correctly because of high contribution of "fakes". It is considered as a task of utmost importance for the experimental data processing. In addition, "fake" tracks obtained from "fake" hits are filtered out using some criteria in course of tracking procedure.

\subsection{Realistic simulations}

The data obtained from Monte Carlo simulations when charged particles are passed through the geometry volumes using GEANT3/4 do not take into account specific aspect concerning the formation of signals in a detector setup. A simplified interpretation, e.g. Monte Carlo points describing exact positions of tracks without "fake" production and other effects, is considered. It looks sufficient to get some basic characteristics of a detector, but it is not appropriate to describe and take into account a list of realistic effects taking place when processing real experimental data.

In order to go towards a realistic simulation of a detector we developed a program model, applicable to the GEM detector, that uses information got from the Garfield++ simulations [2]. These simulations use geometry characteristics of the GEM detector (distances between amplifying gaps), magnitude and orientation of electric and magnetic fields inside the gaps, composition of gas mixture as input. After the simulations performed, we have a possibility to produce clusters on readout plane with characteristics similar to those that we obtain in the experimental setup.

The BM@N setup uses "thick" GEMs with a typical value of width close to $9 \mathrm{~mm}$. Since they are located in an inhomogeneous magnetic field, it is necessary to take into account its influence. Due to the indicated width noticeable shifts of electrons by the Lorentz force take place. It leads to a biased position of clusters registered in the readout plane. For a gas mixture used in the last experimental runs $\left(\mathrm{ArCO}_{2}(70: 30)\right)$ at given magnitudes of electric 
field inside the amplifying gaps (1000:2500:3750:6300 V/cm) and magnetic field (0.9 T) an average shift of cluster is of order of $1.7 \mathrm{~mm}$.

All obtained parameters derived from simulations allowed us to calculate and take into account shifts as a function of magnetic field caused by the Lorentz force when experimental data processing. Due to this, the efficiency of reconstruction of real coordinates of hits has been essentially increased.

\section{Tracking}

An algorithm based on cellular automaton [3] and integrated in the BmnRoot software [4] is used to reconstruct parameters of tracks of charged particles. In this approach, a cell is defined and considered as a line segment connecting two hits pertaining to different planes of the inner tracker. The algorithm consists of the following steps:

1. Creation of cells. At this stage, all possible connections between adjacent detector planes are created. A restriction on a maximal value of the slope in ZX and ZY planes is used when creating cells.

2. Calculation of cell states. The stage is realized iteratively. At first step all cells have a state equal to unity. Further, in course of iterations, if two cells have a common hit and similar slopes in ZX and ZY planes, then the state of the right cell is increased by unity. The iterations are done over all elements (planes) of the inner tracker. It allows one to calculate the final states for all cells.

3. Creation of candidates to be considered as future tracks. At this stage, the found cells are unified with their left neighbors if the state difference is equal to unity. As a result, an array of candidates to be tracks consisting of few cells is created. A candidate is rejected if having less than four hits. The "kept" candidates are passed through procedures aimed to estimate the vector of state and the covariance matrix by a fit with a circle.

4. Smoothing of parameters of candidates. The candidates are processed by the Kalman filter to improve their parameters found at the previous step.

5. Sort of candidates. The stage is introduced in order to sort the found candidates over the number of hits and $\chi^{2}$-criterion to reject inappropriate candidates still kept at previous steps.

6. Selection of tracks. If two sorted candidates already have a shared hit, then the preference is given to a candidate with a less value of $\chi^{2}$ and a larger number of hits. This candidate being considered from now on as a track, is written to the output array of found tracks.

The algorithm developed by our group can reconstruct curved tracks in a magnetic field as well as straight ones in absence of a magnetic field. A set of quality assurance tests done with the help of the Monte Carlo input representing interactions of an argon beam with a kinetic energy of order of $3.2 \mathrm{AGeV}$ with a lead target showed reasonable values of the tracking efficiency, a good precision of a primary vertex and a momentum reconstruction (see Fig. 4). The obtained average level of efficiency is close to $80 \%$ for a wide range of momentum. In addition, a contribution of the silicon part of the inner tracker looks extremely important when reconstructing primary vertex. It allows improving the vertex resolution by a factor of four. 

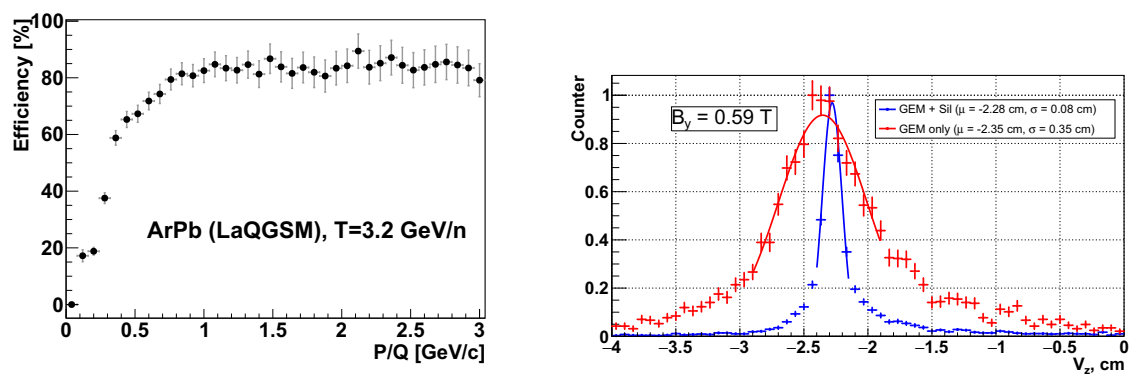

Figure 4. Tracking efficiency and vertex resolution obtained in the quality assurance tests

The algorithm was used to reconstruct tracks in the inner tracker obtained from existing experimental data recorded in the last experimental run. In particular, it was used to perform the track based alignment (Sect. 4) of the inner tracker. As for the recorded data with a magnetic field, Fig. 5 demonstrates the momentum spectra for different targets as a function of number of hits per track. The figure shows the increasing manifestation of spectators and light fragments for lighter targets that is in agreement with theoretical predictions.
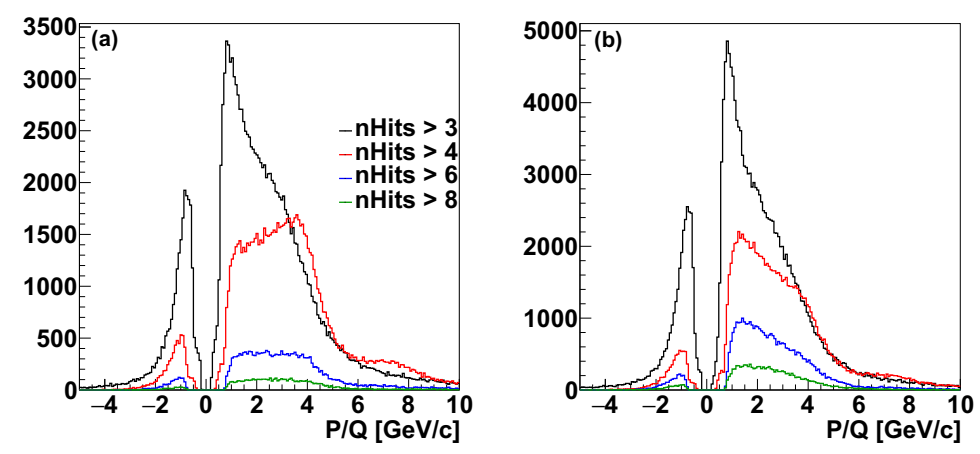

Figure 5. Momentum spectra of reconstructed charged particles for carbon (a) and lead (b) targets as a function of number of hits per track

The distribution of the reconstructed primary vertex is shown in Fig. 6. The primary vertex is calculated using the method of virtual planes. At the moment, the obtained resolution is worse if compared with the results of the Monte Carlo simulation (see Fig. 4), thus challenging further improvements of the algorithm.

\section{Alignment}

The track based alignment is considered as a task of utmost importance. The BmnRoot framework has a well developed software ALCOPACK (ALignment COrrection PACKage) to be used for the alignment of the inner tracker.

The software is based on the Millepede-II formalism [5]. At present, the track model used does not take into account material effects and allows one a simple parameterization by a straight line. More sophisticated track models taking into account a wider set of realistic 


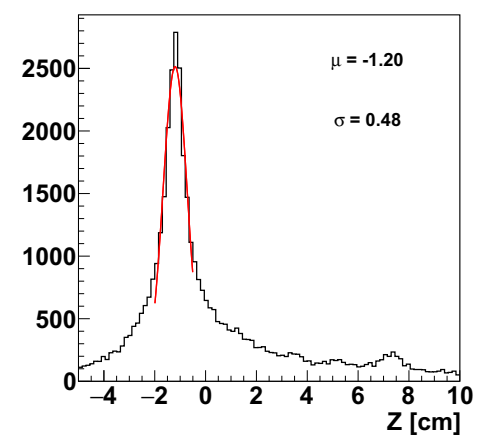

Figure 6. Distribution of $z$ coordinate of the primary vertex. Interactions with the lead target are used for analysis

effects are being tested now. Moreover, the software allows one to include or exclude detectors from the alignment, thus making it possible to align them separately. The mechanism of constraints based on the Lagrange multipliers can be used to prevent a total shift of the detector.

Fig. 7 shows distributions of $z$ coordinate of the primary vertex before and after the alignment done. As a result, the width of the distribution has been reduced by a factor of two.
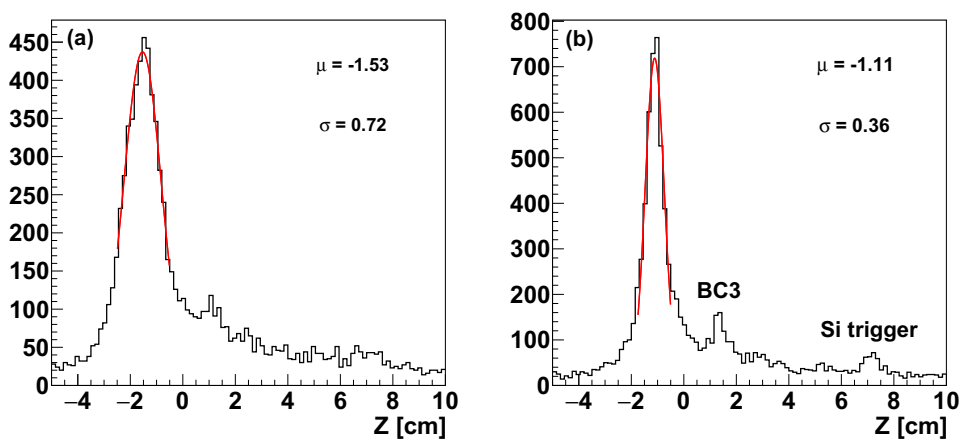

Figure 7. Distribution of $z$ coordinate of the primary vertex before (a) and after (b) the alignment. The used data are recorded without a magnetic field

Positions of some trigger detectors (BC3 and Si trigger) are distinguished better after the alignment done.

Acknowledgments. We thank N. Kutovskiy and his team [6] for an opportunity to use the well equipped JINR cloud infrastructure to perform the calculations, which were done when processing the experimental data. We are grateful to M. Kapishin, N. Zamiatin, E. Zubarev for fruitful discussions and considerations when the work was initiated. 


\section{References}

[1] D. Baranov, M. Kapishin, T. Mamontova, G. Pokatashkin, I. Rufanov, V. Vasendina, and A. Zinchenko, KnE Energ. Phys. 3, 291 (2018)

[2] D. Pfeiffer et al., arXiv:1806.05880 [physics.ins-det]

[3] R. Glattauer, R. Frühwirth, J. Lettenbichler, and W. Mitaroff, arXiv:1202.2761

[4] K. Gertsenberger, S. Merts, O. Rogachevsky et al., Eur. Phys. J. A 52, 214 (2016)

[5] V. Blobel and C. Kleinwort, arXiv:hep-ex/0208021

[6] A.V. Baranov, N.A. Balashov, N.A. Kutovskiy, R.N. Semenov, Physics of Particles and Nuclei Letters 13(5), 672 (2016) 